\title{
Bifurcations of Nontwisted Heteroclinic Loop with Resonant Eigenvalues
}

\author{
Yinlai Jin, ${ }^{1}$ Xiaowei Zhu, ${ }^{1,2}$ Zheng Guo, ${ }^{1}$ Han Xu, ${ }^{1}$ Liqun Zhang, ${ }^{1,2}$ and Benyan Ding ${ }^{1,2}$ \\ ${ }^{1}$ School of Science, Linyi University, Linyi, Shandong 276005, China \\ ${ }^{2}$ School of Mathematics Science, Shandong Normal University, Jinan 250014, China \\ Correspondence should be addressed to Xiaowei Zhu; xiaoweizhu01@sina.com
}

Received 21 January 2014; Accepted 17 March 2014; Published 16 April 2014

Academic Editors: M. Han, Z. Jin, and Y. Xia

Copyright (c) 2014 Yinlai Jin et al. This is an open access article distributed under the Creative Commons Attribution License, which permits unrestricted use, distribution, and reproduction in any medium, provided the original work is properly cited.

By using the foundational solutions of the linear variational equation of the unperturbed system along the heteroclinic orbits to establish the local coordinate systems in the small tubular neighborhoods of the heteroclinic orbits, we study the bifurcation problems of nontwisted heteroclinic loop with resonant eigenvalues. The existence, numbers, and existence regions of 1-heteroclinic loop, 1-homoclinic loop, 1-periodic orbit, 2-fold 1-periodic orbit, and two 1-periodic orbits are obtained. Meanwhile, we give the corresponding bifurcation surfaces.

\section{Introduction and Hypotheses}

With the development of nonlinear science and the deep study of chaotic phenomenon, many experts have been interested in the research on the bifurcation problems of homoclinic and heteroclinic loops for high-dimensional nonlinear dynamical systems (see [1-5] and references therein). Compared with the bifurcations of homoclinic and heteroclinic loops for planer systems, the obtained results have been not rich because of the complexity and the lack of methods. In 1990, Chow et al. studied the codimension 2 homoclinic bifurcation under nondegenerate condition at resonant eigenvalues in [6]. The traditional methods to construct Poincaré maps were adopted to later research. In 1998, Zhu studied the bifurcation problems of nondegenerated homoclinic loops for high-dimensional system $\dot{z}=f(z, \alpha)+$ $\epsilon g(z, \mu, \epsilon)$ in [7] and the bifurcations of nondegenerated heteroclinic loops for high-dimensional system $\dot{z}=f(z)+$ $g(z, \mu)$ in [8]. The methods used in $[7,8]$ were by generalizing the Floquet method to build the local coordinate systems and Poincaré map. The papers $[7,8]$ used the inherent characteristic values to describe the bifurcation surfaces and phenomena so that the results had the practicability and maneuverability.
In [9], the authors studied the bifurcations of rough heteroclinic loops with two saddle point. In [10], Tian and Zhu studied the bifurcations of fine heteroclinic loops with two saddle points. The heteroclinic loops studied in $[9,10]$ were both nontwisted and with no resonant eigenvalues.

In this paper, we study the bifurcation problems of heteroclinic loop with resonant eigenvalues for high-dimensional system $\dot{z}=f(z)+g(z, \mu)$. By simplifying the method in $[7,8]$, we use the foundational solutions of the linear variational equation of the unperturbed system along the heteroclinic orbits to establish the suitable local coordinate systems in the small tubular neighborhoods of the heteroclinic orbits. Then, we get the Poincaré maps and bifurcation equations by means of the improved method.

Consider the $C^{r}$ system

$$
\dot{z}=f(z),
$$

where $r \geq 4, z \in R^{m+n}$. We assume the following.

(H1) Hyperbolic assumption: $z=p_{i}$ are hyperbolic critical points of $(1), i=1,2$. The stable manifold $W_{p_{i}}^{s}$ and the unstable manifold $W_{p_{i}}^{u}$ of $p_{i}$ are $m$-dimensional and $n$-dimensional, respectively. Moreover, $-\rho_{i}^{1}$ and $\lambda_{i}^{1}$ are the simple real eigenvalues of $D_{z} f\left(p_{i}\right)$ such that any other 
eigenvalue $\sigma$ of $D_{z} f\left(p_{i}\right)$ satisfies either $\operatorname{Re} \sigma<-\rho_{i}^{0}<-\rho_{i}^{1}<0$ or $0<\lambda_{i}^{1}<\lambda_{i}^{0}<\operatorname{Re} \sigma$, where $\rho_{i}^{0}$ and $\lambda_{i}^{0}$ are some positive constants.

(H2) Nondegeneration: system (1) has a heteroclinic loop $\Gamma=\Gamma_{1} \cup \Gamma_{2}$, where $\Gamma_{i}=\left\{z=r_{i}(t): t \in R\right\}, r_{i}(+\infty)=$ $r_{i+1}(-\infty)=p_{i+1}, r_{3}(t)=r_{1}(t), p_{3}=p_{1} \cdot \operatorname{dim}\left(T_{r_{i}(t)} W_{p_{i}}^{u} \cap\right.$ $\left.T_{r_{i}(t)} W_{p_{i+1}}^{s}\right)=1$.

(H3) Strong inclination: $\lim _{x \rightarrow+\infty}\left(T_{r_{i}(t)} W_{p_{i}}^{u}+\right.$ $\left.T_{r_{i}(t)} W_{p_{i+1}}^{s}\right)=T_{p_{i+1}} W_{p_{i+1}}^{u u} \oplus T_{p_{i+1}} W_{p_{i+1}}^{s}, \lim _{x \rightarrow-\infty}\left(T_{r_{i}(t)} W_{p_{i}}^{u}+\right.$ $\left.T_{r_{i}(t)} W_{p_{i+1}}^{s+1}\right)=T_{p_{i}} W_{p_{i}}^{u} \oplus T_{p_{i}} W_{p_{i}}^{s s}$, where $W_{p_{i}}^{u \mathcal{u}}$ and $W_{p_{i}}^{s s}$ are the strong unstable manifolds and the strong stable manifolds, respectively, $T_{p_{i}} W_{p_{i}}^{u u}$ is the generalized eigenspace corresponding to all the eigenvalues with larger real part than $\lambda_{i}^{0}$, and $T_{p_{i}} W_{p_{i}}^{s s}$ is the generalized eigenspace corresponding to all the eigenvalues with smaller real part than $-\rho_{i}^{0}$. Denote $e_{i}^{ \pm}=\lim _{t \rightarrow \mp \infty} \dot{r}_{i}(t) /\left|\dot{r}_{i}(t)\right|$, where $e_{i}^{+} \in T_{p_{i}} W_{p_{i}}^{u}$ and $e_{i}^{-} \in T_{p_{i+1}} W_{p_{i+1}}^{s}$ are unit eigenvectors corresponding to $\lambda_{i}^{1}$ and $-\rho_{i+1}^{1}$, respectively. Furthermore, $\operatorname{span}\left(T_{p_{i}} W_{p_{i}}^{u u}, e_{i}^{+}\right)=T_{p_{i}} W_{p_{i}}^{u}$, $\operatorname{span}\left(T_{p_{i}} W_{p_{i}}^{s s}, e_{i+1}^{-}\right)=T_{p_{i}} W_{p_{i}}^{s}$.

Now, we consider the following $C^{r}$ system:

$$
\dot{z}=f(z)+g(z, \mu),
$$

where $\mu \in R^{l}, l \geq 2,0 \leq|\mu| \ll 1, g\left(p_{i}, \mu\right)=g(z, 0)=0$, $i=1,2$.

\section{Local Coordinate Systems}

Suppose that $U_{i}$ is a sufficiently small neighborhood of $z=p_{i}$ and $(\mathrm{H} 1) \sim(\mathrm{H} 3)$ hold; then, for $|\mu|$ small enough, there exists a $C^{r}$ transformation such that system (2) has the following form in $U_{i}$, respectively:

$$
\begin{aligned}
& \dot{x}=\left[\lambda_{i}^{1}(\mu)+\cdots\right] x+\text { h.o.t., } \\
& \dot{y}=\left[-\rho_{i}^{1}(\mu)+\cdots\right] y+\text { h.o.t., } \\
& \dot{u}=\left[B_{i}^{1}(\mu)+\cdots\right] u+\text { h.o.t., } \\
& \dot{v}=\left[-B_{i}^{2}(\mu)+\cdots\right] v+\text { h.o.t., }
\end{aligned}
$$

where $z=\left(x, y, u^{*}, v^{*}\right)^{*}, x \in R^{1}, y \in R^{1}, u \in R^{n-1}, v \in R^{m-1}$, * means transposition, $\lambda_{i}^{1}(0)=\lambda_{i}^{1}, \rho_{i}^{1}(0)=\rho_{i}^{1}, \operatorname{Re} \sigma\left(B_{i}^{1}(0)\right)>$ $\lambda_{i}^{0}$, and $\operatorname{Re} \sigma\left(-B_{i}^{2}(0)\right)<-\rho_{i}^{0}$. Moreover, we suppose

$$
\begin{aligned}
W_{p_{i}}^{u} & =\{z: y=0, v=0\}, \\
W_{p_{i}}^{s} & =\{z: x=0, u=0\}, \\
W_{p_{i}}^{u u} & =\{z: x=x(u), y=0, v=0\}, \\
W_{p_{i}}^{s s} & =\{z: x=0, u=0, y=y(v)\}, \\
\Gamma \cap W_{p_{i}}^{u} & =\{z: u=u(x), y=0, v=0\}, \\
\Gamma \cap W_{p_{i}}^{s} & =\{z: x=0, u=0, v=v(y)\},
\end{aligned}
$$

where $x(0)=\dot{x}(0)=0, y(0)=\dot{y}(0)=0, u(0)=\dot{u}(0)=0$, $v(0)=\dot{v}(0)=0$.

Let $r_{i}(t)=\left(r_{i}^{x}(t), r_{i}^{y}(t),\left(r_{i}^{u}(t)\right)^{*},\left(r_{i}^{v}(t)\right)^{*}\right)^{*}$; we can select some times $T_{i}^{0}$ and $T_{i}^{1}$ such that $r_{i}\left(-T_{i}^{0}\right)=$ $\left(\delta, 0, \delta_{u}^{*}, 0^{*}\right)^{*}, r_{i}\left(T_{i}^{1}\right)=\left(0, \delta, 0^{*}, \delta_{v}^{*}\right)^{*}$, where $\delta$ is small enough such that $\left\{\left(x, y, u^{*}, v^{*}\right)^{*}:|x|,|y|,|u|,|v|<\right.$ $2 \delta\} \subset U_{i},\left|\delta_{u}\right|=O\left(\delta^{\omega}\right),\left|\delta_{v}\right|=O\left(\delta^{\omega}\right), \omega=$ $\min \left\{\operatorname{Re} \sigma\left(B_{i}^{2}(\mu)\right) / \rho_{i}^{1}(\mu), \operatorname{Re} \sigma\left(B_{i}^{1}(\mu)\right) / \lambda_{i}^{1}(\mu)\right\}>1$.

Consider the linear variational system and its adjoint system

$$
\begin{aligned}
& \dot{z}=D f\left(r_{i}(t)\right) z, \\
& \dot{\phi}=-\left(D f\left(r_{i}(t)\right)\right)^{*} \phi .
\end{aligned}
$$

By $[8,9,11-13]$, system (5) and (6) have exponential dichotomies in $R^{+}$and $R^{-}$. It follows from [4] that system (5) has a fundamental solution matrix $Z_{i}(t)=$ $\left(z_{i}^{1}(t), z_{i}^{2}(t), z_{i}^{3}(t), z_{i}^{4}(t)\right)$, satisfying

$$
\begin{gathered}
z_{i}^{1}(t) \in\left(T_{r_{i}(t)} W_{p_{i}}^{u} \cup T_{r_{i}(t)} W_{p_{i+1}}^{s}\right)^{c}, \\
z_{i}^{2}(t)=-\frac{\dot{r}_{i}(t)}{\left|\dot{r}_{i}^{y}\left(T_{i}^{1}\right)\right|} \in T_{r_{i}(t)} W_{p_{i}}^{u} \cap T_{r_{i}(t)} W_{p_{i+1}}^{s}, \\
z_{i}^{3}(t)=\left(z_{i}^{3,1}(t), \ldots, z_{i}^{3, n-1}(t)\right) \in T_{r_{i}(t)} W_{p_{i}}^{u} \cap\left(T_{r_{i}(t)} W_{p_{i+1}}^{s}\right)^{c} \\
=T_{r_{i}(t)} W_{p_{i}}^{u u}, \\
z_{i}^{4}(t)=\left(z_{i}^{4,1}(t), \ldots, z_{i}^{4, m-1}(t)\right) \in\left(T_{r_{i}(t)} W_{p_{i}}^{u}\right)^{c} \cap T_{r_{i}(t)} W_{p_{i+1}}^{s} \\
=T_{r_{i}(t)} W_{p_{i+1}}^{s s}, \\
Z_{i}\left(-T_{i}^{0}\right)=\left(\begin{array}{cccc}
w_{i}^{11} & w_{i}^{21} & 0 & w_{i}^{41} \\
w_{i}^{12} & 0 & 0 & w_{i}^{42} \\
w_{i}^{13} & w_{i}^{23} & I & w_{i}^{43} \\
0 & 0 & 0 & w_{i}^{44}
\end{array}\right), \\
Z_{i}\left(T_{i}^{1}\right)=\left(\begin{array}{cccc}
1 & 0 & w_{i}^{31} & 0 \\
0 & 1 & w_{i}^{32} & 0 \\
0 & 0 & w_{i}^{33} & 0 \\
w_{i}^{14} & w_{i}^{24} & w_{i}^{34} & I
\end{array}\right),
\end{gathered}
$$

where $w_{i}^{21}<0, \quad w_{i}^{12} \neq 0,\left\|w_{i}^{33}\right\| \neq 0, \quad\left\|w_{i}^{44}\right\| \neq 0$, and $\left|w_{i}^{1 j}\left(w_{i}^{12}\right)^{-1}\right| \ll 1, j \neq 2 ;\left|w_{i}^{2 j}\left(w_{i}^{21}\right)^{-1}\right| \ll 1, j=3,4 ;$ $\left|w_{i}^{3 j}\left(w_{i}^{33}\right)^{-1}\right| \ll 1, j \neq 3 ;\left|w_{i}^{4 j}\left(w_{i}^{44}\right)^{-1}\right| \ll 1, j \neq 4$ for $\delta$ small enough.

Denote $\Phi_{i}(t)=\left(\phi_{i}^{1}(t), \phi_{i}^{2}(t), \phi_{i}^{3}(t), \phi_{i}^{4}(t)\right)=\left(Z_{i}^{-1}(t)\right)^{*} ;$ then $\Phi_{i}(t)$ is a fundamental solution matrix of (6). It is well known that $\phi_{i}^{1}(t)$ is bounded and tends to zero exponentially as $t \rightarrow \pm \infty[8,9,12]$.

Denote $\Delta_{i}=w_{i}^{12} /\left|w_{i}^{12}\right| . \Gamma_{i}$ is nontwisted if $\Delta_{i}=1$ and twisted if $\Delta_{i}=-1$. In this paper, we study the bifurcation problems under the nontwisted condition. 


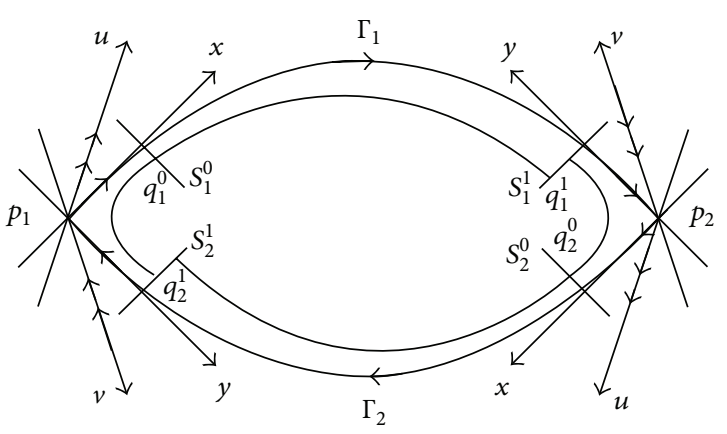

FIGURE 1

Let $z_{i}^{1}(t), z_{i}^{2}(t), z_{i}^{3}(t), z_{i}^{4}(t)$ be the local coordinate systems along $\Gamma_{i}$ in the tubular neighborhood of $\Gamma_{i}$.

\section{Poincaré Maps and Bifurcation Equations}

Denote $h_{i}(t)=r_{i}(t)+Z_{i}(t) N_{i}, N_{i}=\left(n_{i}^{1}, 0,\left(n_{i}^{3}\right)^{*},\left(n_{i}^{4}\right)^{*}\right)^{*}$, $n_{i}^{3}=\left(n_{i}^{3,1}, \ldots, n_{i}^{3, n-1}\right)^{*}, n_{i}^{4}=\left(n_{i}^{4,1}, \ldots, n_{i}^{4, m-1}\right)^{*}$. Define the Poincaré sections of $\Gamma_{i}$ at $t=-T_{i}^{0}$ and $t=T_{i}^{1}$ by

$$
\begin{aligned}
& S_{i}^{0}=\left\{z=h_{i}\left(-T_{i}^{0}\right):|x|,|y|,|u|,|v|<2 \delta\right\}, \\
& S_{i}^{1}=\left\{z=h_{i}\left(T_{i}^{1}\right):|x|,|y|,|u|,|v|<2 \delta\right\},
\end{aligned}
$$

respectively, where $\delta$ is small enough such that $S_{i}^{0} \subset U_{i}, S_{i}^{1} \subset$ $U_{i+1}, U_{3}=U_{1}$ (Figure 1).

Now, we set up the Poincaré map $F_{i}=F_{i}^{1} \circ F_{i}^{0}: S_{i-1}^{1} \mapsto S_{i}^{1}$, where $F_{i}^{0}: q_{i-1}^{1} \in S_{i-1}^{1} \mapsto q_{i}^{0} \in S_{i}^{0}, F_{i}^{1}: q_{i}^{0} \in S_{i}^{0} \mapsto q_{i}^{1} \in S_{i}^{1}$, $S_{0}^{1}=S_{2}^{1}$, and $q_{0}^{1}=q_{2}^{1}$. Here, $F_{i}^{1}$ is constructed from the flow of (2) in the small tubular neighborhood of $\Gamma_{i} ; F_{i}^{0}$ is induced by the flow of the linear approximate system of (3) in the small neighborhood $U_{i}$ of $p_{i}$.

Denote

$$
\begin{aligned}
& q_{i}^{0}=\left(x_{i}^{0}, y_{i}^{0},\left(u_{i}^{0}\right)^{*},\left(v_{i}^{0}\right)^{*}\right)^{*}=r_{i}\left(-T_{i}^{0}\right)+Z_{i}\left(-T_{i}^{0}\right) N_{i}^{0}, \\
& q_{i}^{1}=\left(x_{i}^{1}, y_{i}^{1},\left(u_{i}^{1}\right)^{*},\left(v_{i}^{1}\right)^{*}\right)^{*}=r_{i}\left(T_{i}^{1}\right)+Z_{i}\left(T_{i}^{1}\right) N_{i}^{1},
\end{aligned}
$$

where $N_{i}^{0}=\left(n_{i}^{0,1}, 0,\left(n_{i}^{0,3}\right)^{*},\left(n_{i}^{0,4}\right)^{*}\right)^{*}, N_{i}^{1}=\left(n_{i}^{1,1}, 0,\left(n_{i}^{1,3}\right)^{*}\right.$, $\left.\left(n_{i}^{1,4}\right)^{*}\right)^{*}$.

By the expressions of $Z_{i}\left(-T_{i}^{0}\right)$ and $Z_{i}\left(T_{i}^{1}\right)$, we get that the relations of the two kinds coordinates of $q_{i}^{j}(j=0,1)$ are $x_{i}^{0} \approx$ $\delta, y_{i}^{1} \approx \delta$, and

$$
\begin{aligned}
n_{i}^{0,1}= & \left(w_{i}^{12}\right)^{-1}\left[y_{i}^{0}-w_{i}^{42}\left(w_{i}^{44}\right)^{-1} v_{i}^{0}\right], \\
n_{i}^{0,3}= & u_{i}^{0}-\delta_{u}+\left[w_{i}^{11} w_{i}^{23}\left(w_{i}^{21}\right)^{-1}-w_{i}^{13}\right]\left(w_{i}^{12}\right)^{-1} y_{i}^{0} \\
+ & \left\{w_{i}^{13}\left(w_{i}^{12}\right)^{-1} w_{i}^{42}-w_{i}^{43}-w_{i}^{23}\left(w_{i}^{21}\right)^{-1}\right. \\
& \left.\times\left[w_{i}^{11}\left(w_{i}^{12}\right)^{-1} w_{i}^{42}-w_{i}^{41}\right]\right\}\left(w_{i}^{44}\right)^{-1} v_{i}^{0},
\end{aligned}
$$

$$
\begin{gathered}
n_{i}^{0,4}=\left(w_{i}^{44}\right)^{-1} v_{i}^{0}, \\
n_{i}^{1,1}=x_{i}^{1}-w_{i}^{31}\left(w_{i}^{33}\right)^{-1} u_{i}^{1}, \\
n_{i}^{1,3}=\left(w_{i}^{33}\right)^{-1} u_{i}^{1}, \\
n_{i}^{1,4}=-w_{i}^{14} x_{i}^{1}+\left(w_{i}^{14} w_{i}^{31}+w_{i}^{24} w_{i}^{32}-w_{i}^{34}\right)\left(w_{i}^{33}\right)^{-1} u_{i}^{1} \\
+v_{i}^{1}-\delta_{v} .
\end{gathered}
$$

First, we built the map $F_{i}^{1}: S_{i}^{0} \mapsto S_{i}^{1}, q_{i}^{0} \mapsto q_{i}^{1}$. We make a coordinate transformation $z=h_{i}(t), t \in\left[-T_{i}^{0}, T_{i}^{1}\right]$ and substitute it into (2). By $\dot{r}_{i}(t)=f\left(r_{i}(t)\right), \dot{Z}_{i}(t)=$ $D f\left(r_{i}(t)\right) Z_{i}(t)$ and $\Phi_{i}^{*}(t) Z_{i}(t)=I ;(2)$ becomes

$$
\dot{n}_{i}^{j}=\phi_{i}^{j^{*}}(t) g_{\mu}\left(r_{i}(t), 0\right) \mu+\text { h.o.t., } \quad j=1,3,4 .
$$

Thus, we define $F_{i}^{1}: S_{i}^{0} \mapsto S_{i}^{1}, q_{i}^{0} \mapsto q_{i}^{1}$ as

$$
n_{i}^{j}\left(T_{i}^{1}\right)=n_{i}^{j}\left(-T_{1}^{0}\right)+M_{i}^{j} \mu+\text { h.o.t., } \quad j=1,3,4 .
$$

That is,

$$
n_{i}^{1, j}=n_{i}^{0, j}+M_{i}^{j} \mu+\text { h.o.t., } \quad j=1,3,4,
$$

where $M_{i}^{j}=\int_{-T^{0}}^{T_{i}^{1}} \phi_{i}^{j^{*}}(t) g_{\mu}\left(r_{i}(t), 0\right) d t, i=1,2, j=1,3,4$, are called the Melnikov vectors.

By $[7-9,14], M_{i}^{j}=\int_{-T_{i}^{0}}^{T_{1}^{1}} \phi_{i}^{j^{*}}(t) g_{\mu}\left(r_{i}(t), 0\right) d t=$ $\int_{-\infty}^{+\infty} \phi_{i}^{j^{*}}(t) g_{\mu}\left(r_{i}(t), 0\right) d t, i=1,2, j=1,3,4$.

Second, we built the map $F_{i}^{0}: S_{i-1}^{1} \mapsto S_{i}^{0}, q_{i-1}^{1} \mapsto q_{i}^{0}, S_{0}^{1}=$ $S_{2}^{1}, q_{0}^{1}=q_{2}^{1}$. Without loss of generality, we assume $\rho_{i}^{1} \geq \lambda_{i}^{1}$. Let $\tau_{i}$ be the flying time from $q_{i-1}^{1}$ to $q_{i}^{0}$; we say $s_{i}=e^{-\lambda_{i}^{1}(\mu) \tau_{i}}$ is Silnikov time. By (3) and neglecting the higher order terms, we have

$$
\begin{aligned}
& x_{i-1}^{1} \approx s_{i} x_{i}^{0}, \quad y_{i}^{0} \approx s_{i}^{\rho_{i}^{1}(\mu) / \lambda_{i}^{1}(\mu)} y_{i-1}^{1}, \\
& u_{i-1}^{1} \approx s_{i}^{B_{i}^{1}(\mu) / \lambda_{i}^{1}(\mu)} u_{i}^{0}, \quad v_{i}^{0} \approx s_{i}^{B_{i}^{2}(\mu) / \lambda_{i}^{1}(\mu)} v_{i-1}^{1} .
\end{aligned}
$$

Here, $\left(s_{i}, u_{i}^{0}, v_{i-1}^{1}\right)$ is called the Silnikov coordinate.

Last, it follows from (10) (14) that we get the expression of Poincaré map $F_{i}=F_{i}^{1} \circ F_{i}^{0}$ as follows:

$$
\begin{aligned}
& n_{i}^{1,1}=\left(w_{i}^{12}\right)^{-1} \delta s_{i}^{\rho_{i}^{1}(\mu) / \lambda_{i}^{1}(\mu)}+M_{i}^{1} \mu+\text { h.o.t., } \\
& n_{i}^{1,3}=u_{i}^{0}-\delta_{u}+\left[w_{i}^{11} w_{i}^{23}\left(w_{i}^{21}\right)^{-1}-w_{i}^{13}\right]\left(w_{i}^{12}\right)^{-1} \delta s_{i}^{\rho_{i}^{1}(\mu) / \lambda_{i}^{1}(\mu)} \\
& +M_{i}^{3} \mu+\text { h.o.t., } \\
& n_{i}^{1,4}=\left(w_{i}^{44}\right)^{-1} s_{i}^{B_{i}^{2}(\mu) / \lambda_{i}^{1}(\mu)} v_{i-1}^{1}+M_{i}^{4} \mu+\text { h.o.t. }
\end{aligned}
$$


Denote $G_{i}\left(q_{i-1}^{1}\right)=\left(G_{i}^{1}, G_{i}^{3}, G_{i}^{4}\right)=F_{i}\left(q_{i-1}^{1}\right)-q_{i}^{1}$. By $(10)$ and (15), we get the successor function $G_{i}$ as follows:

$$
\begin{aligned}
& G_{i}^{1}=\delta {\left[\left(w_{i}^{12}\right)^{-1} s_{i}^{\rho_{i}^{1}(\mu) / \lambda_{i}^{1}(\mu)}-s_{i+1}\right]+M_{i}^{1} \mu+\text { h.o.t., } } \\
& G_{i}^{3}=u_{i}^{0}-\delta_{u}+\left[w_{i}^{11} w_{i}^{23}\left(w_{i}^{21}\right)^{-1}-w_{i}^{13}\right]\left(w_{i}^{12}\right)^{-1} \delta s_{i}^{\rho_{i}^{1}(\mu) / \lambda_{i}^{1}(\mu)} \\
& \quad\left(w_{i}^{33}\right)^{-1} s_{i+1}^{B_{i+1}^{1}(\mu) / \lambda_{i+1}^{1}(\mu)} u_{i+1}^{0}+M_{i}^{3} \mu+\text { h.o.t., } \\
& G_{i}^{4}=-v_{i}^{1}+\delta_{v}+w_{i}^{14} \delta s_{i+1}+\left(w_{i}^{44}\right)^{-1} s_{i}^{B_{i}^{2}(\mu) / \lambda_{i}^{1}(\mu)} v_{i-1}^{1} \\
&+M_{i}^{4} \mu+\text { h.o.t. }
\end{aligned}
$$

Let

$$
\left(G_{1}^{1}, G_{1}^{3}, G_{1}^{4}, G_{2}^{1}, G_{2}^{3}, G_{2}^{4}\right)=0 .
$$

Equation (17) is called the bifurcation equation.

\section{The Preservations of Heteroclinic Orbits and Bifurcations of Homoclinic Loops}

In this section, we discuss the nontwisted heteroclinic loop with resonant eigenvalues. Without loss of generality, we assume the following. 1.

(H4) Resonant condition: $\beta_{1}=\rho_{1}^{1} / \lambda_{1}^{1}>1, \beta_{2}=\rho_{2}^{1} / \lambda_{2}^{1}=$

Let $\beta_{i}(\mu)=\rho_{i}^{1}(\mu) / \lambda_{i}^{1}(\mu), \beta_{i}=\beta_{i}(0), \rho_{2}^{1}(\mu)=(1+$ $\alpha(\mu)) \lambda_{2}^{1}(\mu)$. By (H4) and the continuity of function, we get $\beta_{1}(\mu)>1, \beta_{2}(\mu)=1+\alpha(\mu),|\alpha(\mu)| \ll 1,|\alpha(0)|=0$, and $\beta_{1}(\mu) \beta_{2}(\mu)>1$, for $|\mu|=1$.

Consider the solution of (17). Clearly, by the implicit function theorem, the equation $\left(G_{1}^{3}, G_{1}^{4}, G_{2}^{3}, G_{2}^{4}\right)=0$ has a unique solution $u_{i}^{0}=u_{i}^{0}\left(s_{1}, s_{2}, \mu\right), v_{i}^{1}=v_{i}^{1}\left(s_{1}, s_{2}, \mu\right), i=1,2$. Substituting it into $\left(G_{1}^{1}, G_{2}^{1}\right)=0$, we have

$$
\begin{aligned}
& \delta\left[\left(w_{1}^{12}\right)^{-1} s_{1}^{\beta_{1}(\mu)}-s_{2}\right]+M_{1}^{1} \mu+\text { h.o.t. }=0, \\
& \delta\left[\left(w_{2}^{12}\right)^{-1} s_{2}^{1+\alpha(\mu)}-s_{1}\right]+M_{2}^{1} \mu+\text { h.o.t. }=0 .
\end{aligned}
$$

Equation (18) is equivalent to

$$
\begin{aligned}
& s_{1}=\left(w_{2}^{12}\right)^{-1} s_{2}^{1+\alpha(\mu)}+\delta^{-1} M_{2}^{1} \mu+\text { h.o.t. } \\
& s_{2}=\left(w_{1}^{12}\right)^{-1} s_{1}^{\beta_{1}(\mu)}+\delta^{-1} M_{1}^{1} \mu+\text { h.o.t. }
\end{aligned}
$$

Now, we study the existence of heteroclinic orbits and the 1-homoclinic bifurcations. Denote

$$
\begin{aligned}
& R_{1}^{2}=\left\{\mu: M_{1}^{1} \mu>0, w_{2}^{12} M_{2}^{1} \mu<0,|\mu| \ll 1\right\}, \\
& R_{2}^{1}=\left\{\mu: M_{2}^{1} \mu>0, w_{1}^{12} M_{1}^{1} \mu<0,|\mu| \ll 1\right\} .
\end{aligned}
$$

Theorem 1. Suppose that hypotheses $(\mathrm{H1}) \sim(\mathrm{H} 4)$ are valid, and $\operatorname{rank}\left(M_{1}^{1}, M_{2}^{1}\right)=2$; then one has the following. (i) There exists a $(l-1)$-dimensional surface $L_{i}$ with a normal vector $M_{i}^{1}$ at $\mu=0$ such that (2) has a heteroclinic orbit joining $p_{1}$ and $p_{2}$ near $\Gamma_{i}$ if and only if $\mu \in L_{i},|\mu| \ll 1$, where $i=1,2$. Moreover, (2) has a 1-heteroclinic loop near $\Gamma$ if and only if $\mu \in L_{12}=L_{1} \cap L_{2}$ and $|\mu| \ll 1$, where $L_{12}$ is $(l-2)$-dimensional surface and $0 \in L_{12}$. That is, heteroclinic loop $\Gamma$ is persistent.

(ii) There exists a $(l-1)$-dimensional surface $L_{1}^{2} \subset R_{1}^{2}$ which is tangent to $L_{2}\left(L_{1}\right)$ at $\mu=0$ as $\alpha(\mu)>0(\alpha(\mu)<0)$, such that (2) has a unique 1-homoclinic loop $\Gamma_{1}^{2}$ homoclinic to $p_{1}$ near $\Gamma$ as $\mu \in R_{1}^{2} \cap L_{1}^{2}$ and $|\mu| \ll 1$. Meanwhile, there also exists a $(l-1)$-dimensional surface $L_{2}^{1} \subset R_{2}^{1}$ which is tangent to $L_{1}$ at $\mu=0$ such that (2) has a unique 1-homoclinic loop $\Gamma_{2}^{1}$ homoclinic to $p_{2}$ near $\Gamma$ as $\mu \in R_{2}^{1} \cap L_{2}^{1}$ and $|\mu| \ll 1$.

Proof. (i) If (19) has a solution $s_{1}=s_{2}=0$, then, we have

$$
M_{i}^{1} \mu+\text { h.o.t. }=0, \quad i=1,2 .
$$

If $M_{i}^{1} \neq 0$, then, there exists a $(l-1)$-dimensional surface $L_{i}$ defined by (21) with a normal vector $M_{i}^{1}$ at $\mu=0$ such that the $i$ th equation of (19) has a solution $s_{1}=s_{2}=0$ as $\mu \in L_{i}$ and $|\mu| \ll 1$. That is, $\Gamma_{i}$ is persistent.

If rank $\left(M_{1}^{1}, M_{2}^{1}\right)=2$, then there exists a $(l-2)$ dimensional surface $L_{12}=L_{1} \cap L_{2}$ such that (19) has a solution $s_{1}=s_{2}=0$ as $\mu \in L_{12}$ and $|\mu| \ll 1$. That is, $\Gamma$ is persistent.

(ii) If (19) has a solution $s_{1}=0, s_{2}>0$, then, we have

$$
\begin{gathered}
s_{2}=\delta^{-1} M_{1}^{1} \mu+\text { h.o.t. } \\
\left(\delta^{-1} M_{1}^{1} \mu+\text { h.o.t. }\right)^{1+\alpha(\mu)}=-\delta^{-1} w_{2}^{12} M_{2}^{1} \mu+\text { h.o.t. }
\end{gathered}
$$

If $M_{1}^{1} \mu>0, w_{2}^{12} M_{2}^{1} \mu<0$, then, (22) defined a $(l-1)$ dimensional surface $L_{1}^{2}$ with a normal vector $M_{2}^{1}\left(M_{1}^{1}\right)$ at $\mu=$ 0 as $\alpha(\mu)>0(\alpha(\mu)<0)$, such that (19) has a solution $s_{1}=0$, $s_{2}>0$ as $\mu \in L_{1}^{2}$ and $|\mu| \ll 1$. That is, the system (2) has a 1 -homoclinic orbit to $p_{1}$ near $\Gamma$ for $\mu \in L_{1}^{2}$ and $|\mu| \ll 1$.

(iii) If (19) has a solution $s_{1}>0, s_{2}=0$, then, we have

$$
\begin{gathered}
s_{1}=\delta^{-1} M_{2}^{1} \mu+\text { h.o.t., } \\
\left(\delta^{-1} M_{2}^{1} \mu+\text { h.o.t. }\right)^{\beta_{1}(\mu)}=-\delta^{-1} w_{1}^{12} M_{1}^{1} \mu+\text { h.o.t. }
\end{gathered}
$$

If $M_{2}^{1} \mu>0, w_{1}^{12} M_{1}^{1} \mu<0$, then, (23) defined a $(l-1)$ dimensional surface $L_{2}^{1}$ with a normal vector $M_{1}^{1}$ at $\mu=0$ such that (19) has a solution $s_{1}>0, s_{2}=0$ as $\mu \in L_{2}^{1}$ and $|\mu| \ll 1$. That is, the system (2) has a 1 -homoclinic orbit to $p_{2}$ near $\Gamma$ for $\mu \in L_{2}^{1}$ and $|\mu| \ll 1$.

\section{The Periodic Orbits Bifurcations}

Next, we consider the bifurcation problems of 1-periodic orbits near $\Gamma$. In other words, we study the solutions of (19) satisfying $s_{1}>0, s_{2}>0$. We assume the following.

(H5) Nontwisted condition: $\Delta_{1}=\Delta_{2}=1$. 
Obviously, if (H5) holds, we have

$$
\begin{aligned}
& R_{1}^{2}=\left\{\mu: M_{1}^{1} \mu>0, M_{2}^{1} \mu<0,|\mu| \ll 1\right\}, \\
& R_{2}^{1}=\left\{\mu: M_{1}^{1} \mu<0, M_{2}^{1} \mu>0,|\mu| \ll 1\right\} .
\end{aligned}
$$
get

At first, we consider the bifurcations in $R_{1}^{2}$. From (19), we

$$
\begin{aligned}
& \left(s_{1}^{\beta_{1}(\mu)}+\delta^{-1} w_{1}^{12} M_{1}^{1} \mu+\text { h.o.t. }\right)^{1+\alpha(\mu)} \\
& \quad=\left(w_{1}^{12}\right)^{1+\alpha(\mu)} w_{2}^{12}\left(s_{1}-\delta^{-1} M_{2}^{1} \mu+\text { h.o.t. }\right) .
\end{aligned}
$$

Denote $V_{1}\left(s_{1}\right)$ and $N_{1}\left(s_{1}\right)$ are the left and right hands of (25), respectively; then, we have the following.

Lemma 2. Suppose that hypotheses (H1) (H5) are valid; then $V_{1}\left(s_{1}\right)$ is tangent to $N_{1}\left(s_{1}\right)$ at some point $s_{1}$ satisfying $0<s_{1}$, $|\mu| \ll 1$ if and only if $\mu \in R_{1}^{2}, \alpha(\mu)>0,\left|M_{1}^{1} \mu\right| \ll\left|M_{2}^{1} \mu\right|$, and

$$
\begin{aligned}
& \delta^{-1} M_{1}^{1} \mu \\
& =\left(-\frac{M_{1}^{1} \mu}{w_{2}^{12} M_{2}^{1} \mu}\right)^{-1 / \alpha(\mu)}-\left(w_{1}^{12}\right)^{-1} \\
& \quad \times\left(-\frac{w_{1}^{12} M_{1}^{1} \mu}{(1+\alpha(\mu)) \beta_{1}(\mu) M_{2}^{1} \mu}\right)^{\beta_{1}(\mu) /\left(\beta_{1}(\mu)-1\right)} \\
& \quad+\text { h.o.t. }
\end{aligned}
$$

Proof. $V_{1}\left(s_{1}\right)$ is tangent to $N_{1}\left(s_{1}\right)$ at some point $s_{1}$ if and only if $V_{1}\left(s_{1}\right)=N_{1}\left(s_{1}\right)$ and $\dot{V}_{1}\left(s_{1}\right)=\dot{N}_{1}\left(s_{1}\right)$; that is,

$$
\begin{aligned}
& \left(s_{1}^{\beta_{1}(\mu)}+\delta^{-1} w_{1}^{12} M_{1}^{1} \mu+\text { h.o.t. }\right)^{1+\alpha(\mu)} \\
& \quad=\left(w_{1}^{12}\right)^{1+\alpha(\mu)} w_{2}^{12}\left(s_{1}-\delta^{-1} M_{2}^{1} \mu+\text { h.o.t. }\right) \\
& (1+\alpha(\mu)) \beta_{1}(\mu) s_{1}^{\beta_{1}(\mu)-1}\left(s_{1}^{\beta_{1}(\mu)}+\delta^{-1} w_{1}^{12} M_{1}^{1} \mu+\text { h.o.t. }\right)^{\alpha(\mu)} \\
& =\left(w_{1}^{12}\right)^{1+\alpha(\mu)} w_{2}^{12} .
\end{aligned}
$$

So

$$
s_{1}=\left(-\frac{w_{1}^{12} M_{1}^{1} \mu}{(1+\alpha(\mu)) \beta_{1}(\mu) M_{2}^{1} \mu}\right)^{1 /\left(\beta_{1}(\mu)-1\right)}+\text { h.o.t. }
$$

Substituting (28) into the second expression of (27), we have that (26) holds.

Equation (28) means $\mid M_{1}^{1} \mu\|\ll\| M_{2}^{1} \mu \|$, and (26) means $\mu \in R_{1}^{2}$ and $\alpha(\mu)>0$.

If $M_{1}^{1}$ and $M_{2}^{1}$ are linearly independent, then there exists a $(l-1)$-dimensional surface $\widetilde{L}_{1}^{2}$ defined by (26) in the small neighborhood of $\mu=0$. It is easy to know that $\widetilde{L}_{1}^{2} \in R_{1}^{2}$ is tangent to $L_{2}$ for $\alpha(\mu)>0$. By (21), (22), and (26), we get

$$
\begin{aligned}
\left.\delta^{-1} M_{1}^{1} \mu\right|_{L_{1}^{2}}= & \left(-\frac{M_{1}^{1} \mu}{w_{2}^{12} M_{2}^{1} \mu}\right)^{-(1 / \alpha(\mu))}+\text { h.o.t. } \\
> & \left(-\frac{M_{1}^{1} \mu}{w_{2}^{12} M_{2}^{1} \mu}\right)^{-(1 / \alpha(\mu))}-\left(w_{1}^{12}\right)^{-1} \\
& \times\left(-\frac{w_{1}^{12} M_{1}^{1} \mu}{(1+\alpha(\mu)) \beta_{1}(\mu) M_{2}^{1} \mu}\right)^{\beta_{1}(\mu) /\left(\beta_{1}(\mu)-1\right)} \\
& + \text { h.o.t. } \\
= & \left.\delta^{-1} M_{1}^{1} \mu\right|_{\widetilde{L}_{1}^{2}}>0=\left.\delta^{-1} M_{1}^{1} \mu\right|_{L_{1}}
\end{aligned}
$$

That is to say, $\widetilde{L}_{1}^{2}$ is located in the open region between $L_{1}$ and $L_{1}^{2}$. Thus, we have the following.

(1) For $\alpha(\mu)>0$, we define the following three open sections in $R_{1}^{2}:\left(R_{1}^{2}\right)_{1}$ is bounded by $L_{2}$ and $L_{1}^{2},\left(R_{1}^{2}\right)_{2}$ is bounded by $L_{1}^{2}$ and $\widetilde{L}_{1}^{2}$, and $\left(R_{1}^{2}\right)_{0}$ is bounded by $\widetilde{L}_{1}^{2}$ and $L_{1}$, and they have nonempty intersection with $R_{1}^{2}$.

(2) For $\alpha(\mu)<0$, we define the following two open sections in $R_{1}^{2}:\left(R_{1}^{2}\right)_{1}$ is bounded by $L_{2}$ and $L_{1}^{2}$ and $\left(R_{1}^{2}\right)_{0}$ is bounded by $L_{1}^{2}$ and $L_{1}$, and they have nonempty intersection with $R_{1}^{2}$.

Now, we consider the nonnegative solutions of $V_{1}\left(s_{1}\right)=$ $N_{1}\left(s_{1}\right)$ defined by (25). By $V_{1}\left(s_{1}\right)=\left(s_{1}^{\beta_{1}(\mu)}+\delta^{-1} w_{1}^{12} M_{1}^{1} \mu+\right.$ h.o.t. $)^{1+\alpha(\mu)}, N_{1}\left(s_{1}\right)=\left(w_{1}^{12}\right)^{1+\alpha(\mu)} w_{2}^{12}\left(s_{1}-\delta^{-1} M_{2}^{1} \mu+\right.$ h.o.t. $)$, we get $V_{1}(0)=\left(\delta^{-1} w_{1}^{12} M_{1}^{1} \mu+\text { h.o.t. }\right)^{1+\alpha(\mu)}, N_{1}(0)=\left(w_{1}^{12}\right)^{1+\alpha(\mu)}$ $w_{2}^{12}\left(-\delta^{-1} M_{2}^{1} \mu+\right.$ h.o.t.). From (22), $V_{1}(0)=N_{1}(0)$ holds for $\mu \in L_{1}^{2},|\mu| \ll 1$.

By Lemma 2, it is easy to get the following conclusions.

(1) For $\alpha(\mu)>0$, we have the following (see Figure 2).

If $\mu \in\left(R_{1}^{2}\right)_{1}$, then $V_{1}\left(s_{1}\right)=N_{1}\left(s_{1}\right)$ has one small positive solution.

If $\mu \in L_{1}^{2}$, then $V_{1}\left(s_{1}\right)=N_{1}\left(s_{1}\right)$ has one small positive solution and one zero solution.

If $\mu \in\left(R_{1}^{2}\right)_{2}$, then $V_{1}\left(s_{1}\right)=N_{1}\left(s_{1}\right)$ has two small different positive solutions.

If $\mu \in \widetilde{L}_{1}^{2}$, then $V_{1}\left(s_{1}\right)=N_{1}\left(s_{1}\right)$ has one small twofold positive solution.

If $\mu \in\left(R_{1}^{2}\right)_{0}$, then $V_{1}\left(s_{1}\right)=N_{1}\left(s_{1}\right)$ does not have any small nonnegative solutions.

(2) For $\alpha(\mu)<0$, we have the following.

If $\mu \in\left(R_{1}^{2}\right)_{1}$, then $V_{1}\left(s_{1}\right)=N_{1}\left(s_{1}\right)$ has one small positive solution.

If $\mu \in L_{1}^{2}$, then $V_{1}\left(s_{1}\right)=N_{1}\left(s_{1}\right)$ has one zero solution.

If $\mu \in\left(R_{1}^{2}\right)_{0}$, then $V_{1}\left(s_{1}\right)=N_{1}\left(s_{1}\right)$ has not any small nonnegative solutions. 


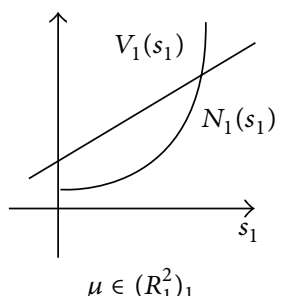

(a)

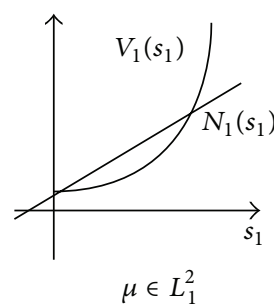

(b)

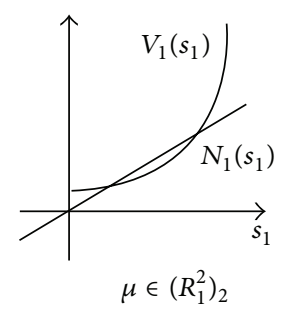

(c)

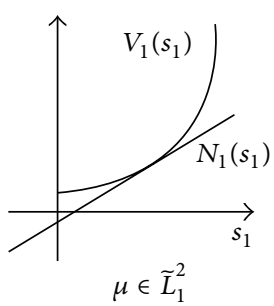

(d)

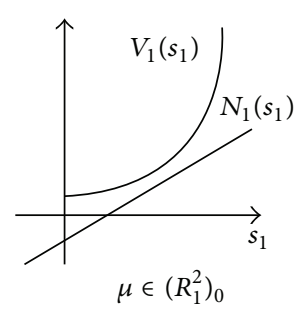

(e)

Figure 2

Thus, we have shown the following conclusions.

Theorem 3. Suppose that hypotheses (H1) (H5) are valid, $\mu \in$ $R_{1}^{2}, \alpha(\mu)>0$; then the following conclusions are true.

(i) System (2) has one simple 1-periodic orbit near $\Gamma$ as $\mu \epsilon$ $\left(R_{1}^{2}\right)_{1}$.

(ii) System (2) has one simple 1-periodic orbit and one 1homoclinic loop homoclinic to $p_{1}$ near $\Gamma$ as $\mu \in L_{1}^{2}$.

(iii) System (2) has two simple 1-periodic orbits near $\Gamma$ as $\mu \in\left(R_{1}^{2}\right)_{2}$.

(iv) System (2) has a unique twofold 1-periodic orbit near Г as $\mu \in \widetilde{L}_{1}^{2}$.

(v) System (2) has not any 1-periodic orbits and 1homoclinic loops near $\Gamma$ as $\mu \in\left(R_{1}^{2}\right)_{0}$.

Theorem 4. Suppose that hypotheses (H1) (H5) are valid, $\mu \in$ $R_{1}^{2}, \alpha(\mu)<0$; then the following conclusions are true.

(i) System (2) has one simple 1-periodic orbit near $\Gamma$ as $\mu \epsilon$ $\left(R_{1}^{2}\right)_{1}$.

(ii) System (2) has one 1-homoclinic loop homoclinic to $p_{1}$ near $\Gamma$ as $\mu \in L_{1}^{2}$.

(iii) System (2) does not have any 1-periodic orbits and 1homoclinic loops near $\Gamma$ as $\mu \in\left(R_{1}^{2}\right)_{0}$.

Next, we consider the bifurcations in $R_{2}^{1}$. Similarly, from (19), we get

$$
\begin{aligned}
& \left(s_{2}^{1+\alpha(\mu)}+\delta^{-1} w_{2}^{12} M_{2}^{1} \mu+\text { h.o.t. }\right)^{\beta_{1}(\mu)} \\
& \quad=w_{1}^{12}\left(w_{2}^{12}\right)^{\beta_{1}(\mu)}\left(s_{2}-\delta^{-1} M_{1}^{1} \mu+\text { h.o.t. }\right) .
\end{aligned}
$$

Denote $V_{2}\left(s_{2}\right)$ and $N_{2}\left(s_{2}\right)$ are the left and right hands of (30), respectively; then one has the following.
Lemma 5. Suppose that hypotheses (H1) (H5) are valid, then $V_{2}\left(s_{2}\right)$ is tangent to $N_{2}\left(s_{2}\right)$ at some point $s_{2}$ satisfying $0<s_{2}$, $|\mu| \ll 1$ if and only if $\alpha(\mu)<0,\left\|M_{1}^{1} \mu\right\| \ll\left\|M_{2}^{1} \mu\right\|, \mu \in R_{2}^{1}$ and

$$
\begin{aligned}
& \delta^{-1} M_{2}^{1} \mu \\
& =\left(-\frac{w_{1}^{12} M_{1}^{1} \mu}{M_{2}^{1} \mu}\right)^{1 /\left(\beta_{1}(\mu)-1\right)}-\left(w_{2}^{12}\right)^{-1} \\
& \quad \times\left(-\frac{(1+\alpha(\mu)) \beta_{1}(\mu) M_{1}^{1} \mu}{w_{2}^{12} M_{2}^{1} \mu}\right)^{-(1+\alpha(\mu)) / \alpha(\mu)} \\
& \quad+\text { h.o.t. }
\end{aligned}
$$

Proof. $V_{2}\left(s_{2}\right)$ is tangent to $N_{2}\left(s_{2}\right)$ at some point $s_{2}$ if and only if $V_{2}\left(s_{2}\right)=N_{2}\left(s_{2}\right)$ and $\dot{V}_{2}\left(s_{2}\right)=\dot{N}_{2}\left(s_{2}\right)$; that is,

$$
\begin{aligned}
& \left(s_{2}^{1+\alpha(\mu)}+\delta^{-1} w_{2}^{12} M_{2}^{1} \mu+\text { h.o.t. }\right)^{\beta_{1}(\mu)} \\
& =w_{1}^{12}\left(w_{2}^{12}\right)^{\beta_{1}(\mu)}\left(s_{2}-\delta^{-1} M_{1}^{1} \mu+\text { h.o.t. }\right), \\
& (1+\alpha(\mu)) \beta_{1}(\mu) s_{2}^{\alpha(\mu)}\left(s_{2}^{1+\alpha(\mu)}+\delta^{-1} w_{2}^{12} M_{2}^{1} \mu+\text { h.o.t. }\right)^{\beta_{1}(\mu)-1} \\
& =w_{1}^{12}\left(w_{2}^{12}\right)^{\beta_{1}(\mu)}+\text { h.o.t. }
\end{aligned}
$$

The solution of (32) is

$$
s_{2}=\left(-\frac{w_{2}^{12} M_{2}^{1} \mu}{(1+\alpha(\mu)) \beta_{1}(\mu) M_{1}^{1} \mu}\right)^{1 / \alpha(\mu)}+\text { h.o.t. }
$$

Substituting (33) into the second expression of (32), we obtain (31).

Equation (33) holds means $\mid M_{1}^{1} \mu\|\ll\| M_{2}^{1} \mu \|, \alpha(\mu)<0$, or $\mid M_{2}^{1} \mu\|\ll\| M_{1}^{1} \mu \|, \alpha(\mu)>0$. But, if $\mid M_{2}^{1} \mu\|\ll\| M_{1}^{1} \mu \|$, $\alpha(\mu)>0$, then, by (31), we get that $\delta^{-1} M_{2}^{1} \mu$ will be tented to $\infty$. Last, $\mu \in R_{2}^{1}$ is obvious.

If $M_{1}^{1}$ and $M_{2}^{1}$ are linearly independent, then there exists a $(l-1)$-dimensional surface $\widetilde{L}_{2}^{1}$ defined by (31) in the small 
neighborhood of $\mu=0$. It is easy to know that $\widetilde{L}_{2}^{1}$ is tangent to $L_{1}$ at $\mu=0$. By (21), (23), and (31), we get

$$
\begin{aligned}
\left.\delta^{-1} M_{2}^{1} \mu\right|_{L_{2}^{1}}= & \left(-\frac{w_{1}^{12} M_{1}^{1} \mu}{M_{2}^{1} \mu}\right)^{1 /\left(\beta_{1}(\mu)-1\right)}+\text { h.o.t. } \\
> & \left(-\frac{w_{1}^{12} M_{1}^{1} \mu}{M_{2}^{1} \mu}\right)^{1 /\left(\beta_{1}(\mu)-1\right)}-\left(w_{2}^{12}\right)^{-1} \\
& \times\left(-\frac{(1+\alpha(\mu)) \beta_{1}(\mu) M_{1}^{1} \mu}{w_{2}^{12} M_{2}^{1} \mu}\right)^{-(1+\alpha(\mu)) / \alpha(\mu)} \\
& + \text { h.o.t. } \\
= & \left.\delta^{-1} M_{2}^{1} \mu\right|_{\widetilde{L}_{2}^{1}}>0=\left.\delta^{-1} M_{2}^{1} \mu\right|_{L_{2}} .
\end{aligned}
$$

That is, $\widetilde{L}_{2}^{1}$ is located in the open region between $L_{2}$ and $L_{2}^{1}$. Thus, we have the following.

(1) For $\alpha(\mu)>0$, we define the following two open sections: $\left(R_{2}^{1}\right)_{1}$ is bounded by $L_{1}$ and $L_{2}^{1}$ and $\left(R_{2}^{1}\right)_{0}$ is bounded by $L_{2}^{1}$ and $L_{2}$, and they have nonempty intersection with $R_{2}^{1}$.

(2) For $\alpha(\mu)<0$, we define the following three open sections: $\left(R_{2}^{1}\right)_{1}$ is bounded by $L_{1}$ and $L_{2}^{1},\left(R_{2}^{1}\right)_{2}$ is bounded by $L_{2}^{1}$ and $\widetilde{L}_{2}^{1}$, and $\left(R_{2}^{1}\right)_{0}$ is bounded by $\widetilde{L}_{2}^{1}$ and $L_{2}$, and they have nonempty intersection with $R_{2}^{1}$.

Now, we consider the nonnegative solutions of $V_{2}\left(s_{2}\right)=$ $N_{2}\left(s_{2}\right)$ defined by (30). By $V_{2}\left(s_{2}\right)=\left(s_{2}^{1+\alpha(\mu)}+\delta^{-1} w_{2}^{12} M_{2}^{1} \mu+\right.$ h.o.t. $)^{\beta_{1}(\mu)}, N_{2}\left(s_{2}\right)=w_{1}^{12}\left(w_{2}^{12}\right)^{\beta_{1}(\mu)}\left(s_{2}-\delta^{-1} M_{1}^{1} \mu+\right.$ h.o.t. $)$, we get $V_{2}(0)=\left(\delta^{-1} w_{2}^{12} M_{2}^{1} \mu+\text { h.o.t. }\right)^{\beta_{1}(\mu)}, N_{2}(0)=w_{1}^{12}\left(w_{2}^{12}\right)^{\beta_{1}(\mu)}$ $\left(-\delta^{-1} M_{1}^{1} \mu+\right.$ h.o.t.). From (23), $V_{2}(0)=N_{2}(0)$ holds for $\mu \in$ $L_{2}^{1},|\mu| \ll 1$.

By Lemma 5, it is easy to get the following conclusions.

(1) For $\alpha(\mu)>0$, we have the following.

If $\mu \in\left(R_{2}^{1}\right)_{1}$, then $V_{2}\left(s_{2}\right)=N_{2}\left(s_{2}\right)$ has one small positive solution.

If $\mu \in L_{2}^{1}$, then $V_{2}\left(s_{2}\right)=N_{2}\left(s_{2}\right)$ has one zero solution.

If $\mu \in\left(R_{2}^{1}\right)_{0}$, then $V_{2}\left(s_{2}\right)=N_{2}\left(s_{2}\right)$ does not have any small nonnegative solutions.

(2) For $\alpha(\mu)<0$, we have the following.

If $\mu \in\left(R_{2}^{1}\right)_{1}$, then $V_{2}\left(s_{2}\right)=N_{2}\left(s_{2}\right)$ has one small positive solution.

If $\mu \in L_{2}^{1}$, then $V_{2}\left(s_{2}\right)=N_{2}\left(s_{2}\right)$ has one small positive solution and one zero solution.

If $\mu \in\left(R_{2}^{1}\right)_{2}$, then $V_{2}\left(s_{2}\right)=N_{2}\left(s_{2}\right)$ has two different positive solutions.

If $\mu \in \widetilde{L}_{2}^{1}$, then $V_{2}\left(s_{2}\right)=N_{2}\left(s_{2}\right)$ has one twofold positive solution.
If $\mu \in\left(R_{2}^{1}\right)_{0}$, then $V_{2}\left(s_{2}\right)=N_{2}\left(s_{2}\right)$ does not have any small nonnegative solutions.

Thus, we have shown the following conclusions.

Theorem 6. Suppose that hypotheses (H1) (H5) are valid, $\mu \in$ $R_{2}^{1}, \alpha(\mu)>0$; then the following conclusions are true.

(i) System (2) has one simple 1-periodic orbit near $\Gamma$ as $\mu \in$ $\left(R_{2}^{1}\right)_{1}$.

(ii) System (2) has one 1-homoclinic loop homoclinic to $p_{2}$ near $\Gamma$ as $\mu \in L_{2}^{1}$.

(iii) System (2) does not have any 1-periodic orbits and 1homoclinic loops near $\Gamma$ as $\mu \in\left(R_{2}^{1}\right)_{0}$.

Theorem 7. Suppose that hypotheses (H1) (H5) are valid, $\mu \in$ $R_{2}^{1}, \alpha(\mu)<0$; then the following conclusions are true.

(i) System (2) has one simple 1-periodic orbit near $\Gamma$ as $\mu \epsilon$ $\left(R_{2}^{1}\right)_{1}$.

(ii) System (2) has one simple 1-periodic orbit and one 1homoclinic loop homoclinic to $p_{2}$ near $\Gamma$ as $\mu \in L_{2}^{1}$.

(iii) System (2) has two simple 1-periodic orbits near $\Gamma$ as $\mu \in\left(R_{2}^{1}\right)_{2}$

(iv) System (2) has a unique twofold 1-periodic orbit near $\Gamma$ as $\mu \in \widetilde{L}_{2}^{1}$.

(v) System (2) does not have any 1-periodic orbits and 1homoclinic loops near $\Gamma$ as $\mu \in\left(R_{2}^{1}\right)_{0}$.

Let $D_{1}^{2}$ be an open region that is bounded by $L_{1}$ and $L_{2}$; meanwhile, $D_{1}^{2} \cap\left\{\mu: M_{1}^{1} \mu>0, M_{2}^{1} \mu>0,|\mu| \ll 1\right\} \neq \emptyset$. Let $D_{2}^{1}$ be an open region that is bounded by $L_{2}$ and $L_{1}$; meanwhile, $D_{2}^{1} \cap\left\{\mu: M_{1}^{1} \mu<0, M_{2}^{1} \mu<0,|\mu| \ll 1\right\} \neq \emptyset$.

By (19), it is easy to see that (19) has an unique solution $s_{1}>0, s_{2}>0$ for $\mu \in D_{1}^{2}$ and does not have any nonnegative solutions for $\mu \in D_{2}^{1}$. So we get the following conclusions.

Theorem 8. Supposing that hypotheses (H1) (H5) are valid, then

(i) system (2) has one simple 1-periodic orbit near $\Gamma$ as $\mu \epsilon$ $D_{1}^{2}$,

(ii) system (2) has not any 1-periodic orbits, 1-homoclinic loops, and heteroclinic orbits near $\Gamma$ as $\mu \in D_{2}^{1}$.

Combining Theorems 1 8, we get the bifurcation figures (see Figures 3 and 4).

\section{Conflict of Interests}

The authors declare that there is no conflict of interests regarding the publication of this paper.

\section{Acknowledgments}

This work is supported by the National Natural Science Foundation of China (no. 10671069), the Natural Science 


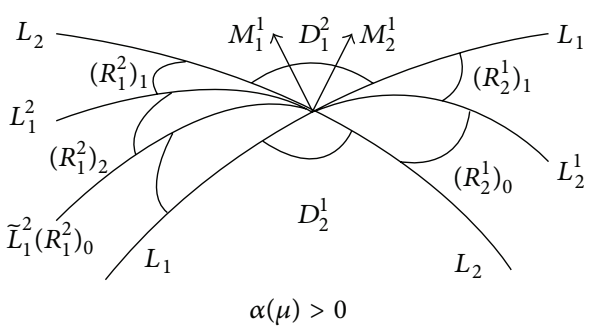

FIGURE 3

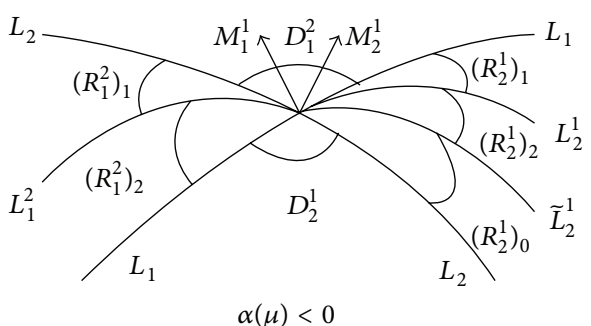

FIGURE 4

Foundation of Shandong Province (no. Y2007A17), and the Applied Mathematics Enhancement Program of Linyi University.

\section{References}

[1] S. N. Chow and J. K. Hale, Methods of Bifurcation Theory, Springer, New York, NY, USA, 1982.

[2] S. Wiggins, Introduction to Applied Nonlinear Dynamical Systems and Chaos, Springer, New York, NY, USA, 1990.

[3] M. Han and D. Zhu, Bifurcation Theory of Differential Equations, Coal Industry Press, Beijing, China, 1994.

[4] Y. Jin and D. Zhu, "Degenerated homoclinic bifurcations with higher dimensions," Chinese Annals of Mathematics B, vol. 21, no. 2, pp. 201-210, 2000.

[5] Y. Jin, F. Li, and H. Xu, "Bifurcations and stability of nondegenerated homoclinic loops for higher dimensional systems," Computational and Mathematical Methods in Medicine, vol. 2013, Article ID 582820, 9 pages, 2013.

[6] S. N. Chow, B. Deng, and B. Fiedler, "Homoclinic bifurcation at resonant eigen- values," Journal of Dynamical Systems and Differential Equations, vol. 2, no. 2, pp. 177-244, 1990.

[7] D. Zhu, "Problems in homoclinic bifurcation with higher dimensions," Acta Mathematica Sinica, vol. 14, no. 3, pp. 341$352,1998$.

[8] D. Zhu and Z. Xia, "Bifurcations of heteroclinic loops," Science in China A: Mathematics, Physics, Astronomy, vol. 41, no. 8, pp. X6-848, 1998.

[9] Y. Jin and D. Zhu, "Bifurcations of rough heteroclinic loop with two saddle points," Science in China A: Mathematics, Physics, Astronomy, vol. 46, no. 4, pp. 159-168, 2003.

[10] Q. Tian and D. Zhu, "Bifurcations of nontwisted heteroclinic loop," Science in China A: Mathematics, Physics, Astronomy, vol. 43, no. 8, pp. 818-828, 2000.

[11] M. Yamashita, "Melnikov vector in higher dimensions," Nonlinear Analysis, vol. 18, no. 7, pp. 657-670, 1992.
[12] D. Zhu, "Melnikov-type vectors and principal normals," Science in China (Scientia Sinica) Series A, vol. 37, no. 7, pp. 814-822, 1994.

[13] D. Zhu, "Exponential trichotomy and heteroclinic bifurcations," Nonlinear Analysis, Theory, Methods and Applications, vol. 28, no. 3, pp. 547-557, 1997.

[14] M. Han, D. Luo, and D. Zhu, "The uniqueness of limit cycles bifurcating from a singular closed orbit (III)," Acta Mathematica Sinica, vol. 35, no. 5, pp. 673-684, 1992. 


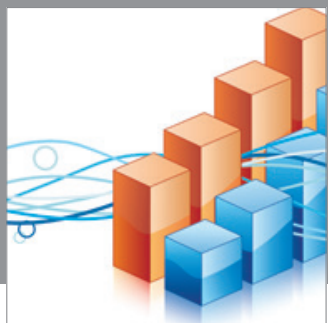

Advances in

Operations Research

mansans

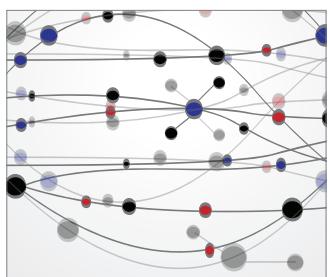

The Scientific World Journal
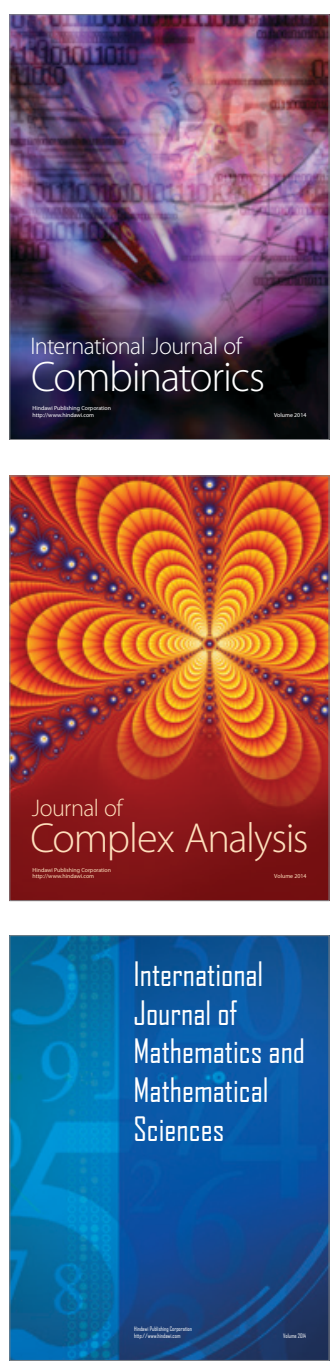
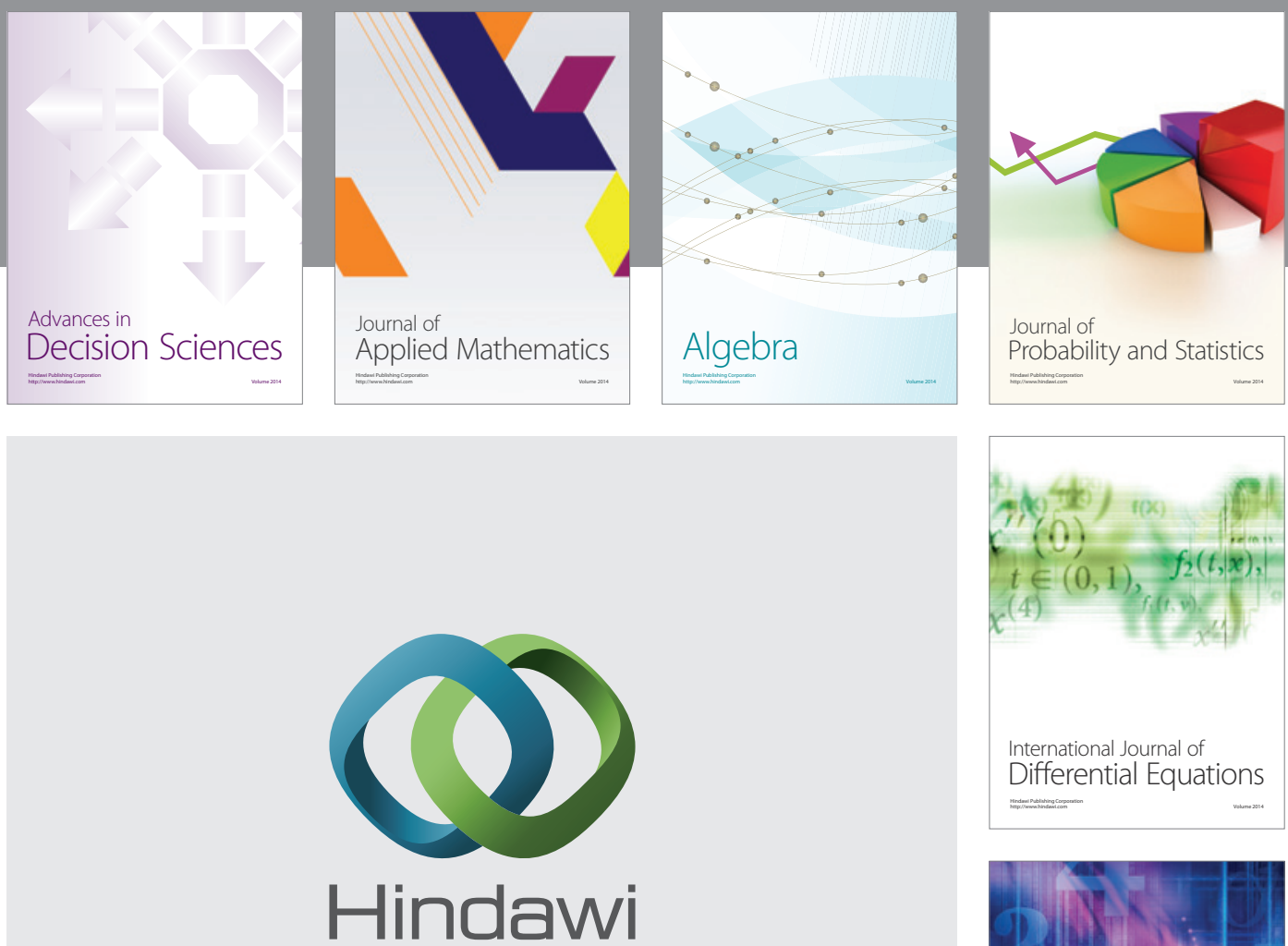

Submit your manuscripts at http://www.hindawi.com
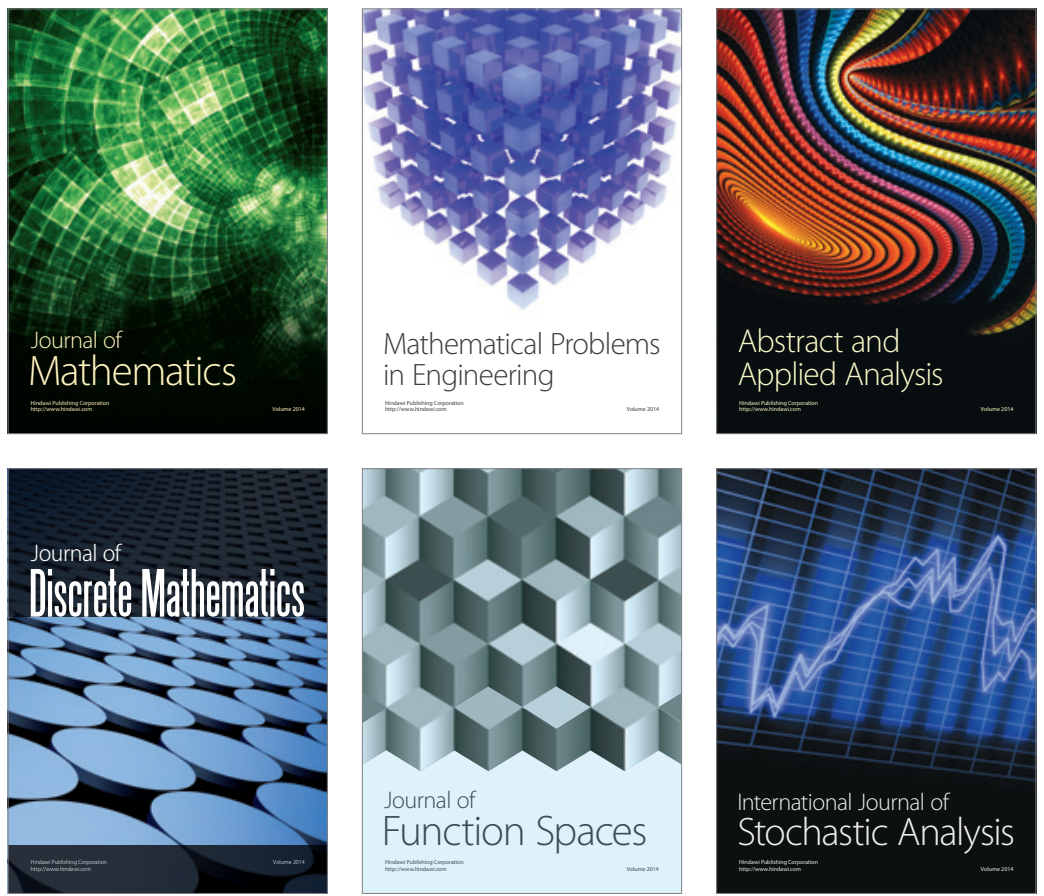

Journal of

Function Spaces

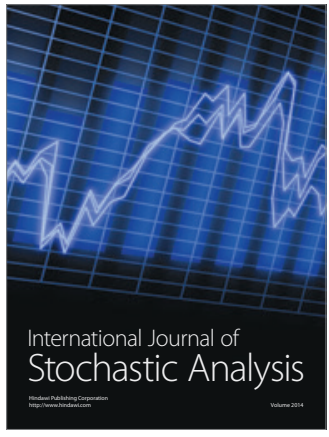

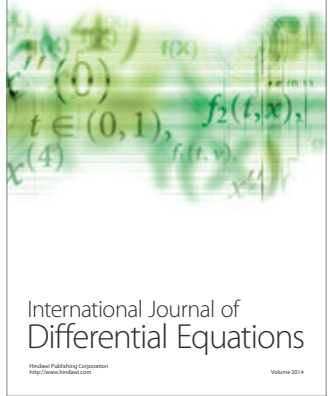
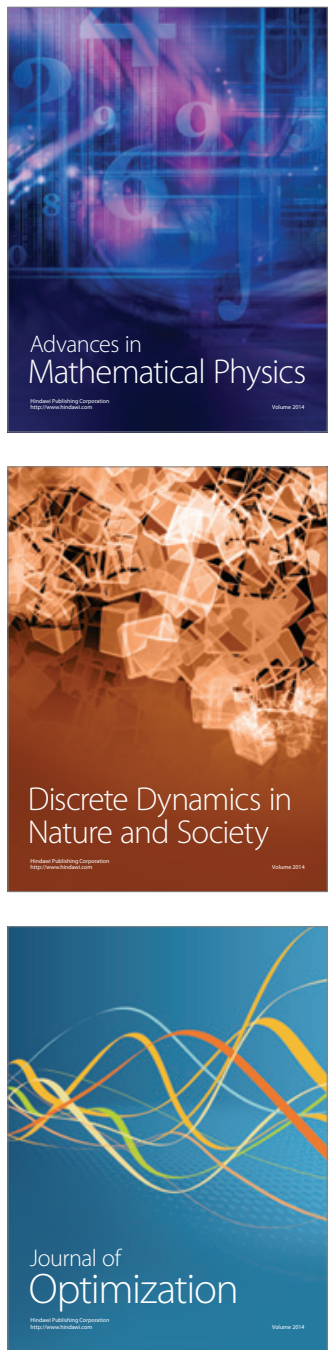